\title{
Investigation of Braking Timing of Drivers for Design of Pedestrian Collision Avoidance System
}

\author{
Keisuke Suzuki $^{1}$, Takuya Kakihara ${ }^{1}$ and Yasutoshi Horii ${ }^{2}$ \\ 1. Faculty of Engineering, Kagawa University, Takamatsu 761-0396, Japan \\ 2. DENSO SALES SWEDEN AB, Göteborg 417 55, Sweden
}

\begin{abstract}
The braking behavior of drivers when a pedestrian comes out from the sidewalk to the road was analyzed using a driving simulator. Based on drivers' braking behavior, the braking control timing of the system for avoiding the collision with pedestrians was proposed. In this study, the subject drivers started braking at almost the same time in terms of TTC (Time to Collision), regardless of the velocity of a subject vehicle and crossing velocity of pedestrians. This experimental result showed that brake timing of the system which can minimize the interference for braking between drivers and the system is $1.3 \mathrm{~s}$ of TTC. Next, the drivers' braking behavior was investigated when the system controlled braking to avoid collision at this timing. As a result, drivers did not show any change of braking behavior with no excessive interference between braking control by the system and braking operation by drivers for avoiding collisions with pedestrians which is equivalent to the excessive dependence on the system.
\end{abstract}

Key words: Active safety, collision avoidance, pedestrian, brake timing, TTC, driving simulator.

\section{Introduction}

The actual utilization and spreading collision-avoidance support brake systems are progressing aiming at preventing rear-end collision to leading vehicle in the market. Regarding the designing of devices, Ishida et al. [1] reported the sensor fusion algorithm to increase the accuracy for detecting the obstacles is an important factor to increase the user-acceptance of the support systems in the market. Miichi et al. [2] also reported the concept of system integration of several active safety technologies named "Integrated Safety Management Concept" for increasing the user and social acceptance of the systems. These are the research activities as a part of ASV (Advanced Safety Vehicle) projects in Japan. In response to the increase of the active safety devices in the market, the evaluation test of collision-avoidance support brake system started in Japan as a part of dynamic vehicle performance test

Corresponding author: Keisuke Suzuki, doctor of engineering, professor, research field: human-machine interface of active safety systems. called J-NCAP in 2014. Regarding J-NCAP, the details of evaluation methods of active safety devices such as AEBS (Autonomous Emergency Braking System) and LDWS (Lane Departure Warning System) were summarized by Ujihashi et al. [3]. There are slight differences in the evaluation methods among J-NCAP, Euro-NCAP and U.S.-NCAP depending on the typical traffic accidents in each region. Though there are slight differences for the evaluation test method, the evaluation of AEBS is progressing because of rapid increase of systems in the market. When we look at the research projects in overseas regarding the system evaluation in terms of collision mitigation of collision-avoidance support brake system, as Distner et al. [4] reported, the City-Safety project in Sweden is one of the famous and well known. For instance, the system was verified using CAE methods and practical tests and it was discussed by Coelingh et al. [5] and Brannstrom et al. [6] what kind of benefit through the utilization of these systems that can be achieved in the real-life safety. In addition to these studies, the studies by Ljung et al. [7] and Isaksson [8] have tried to predict the effect of various 
collision-avoidance support system, and the real world effect of these systems.

For the next phase of spreading the collision-avoidance support brake system in the market, as Inomata et al. [9] and Coelingh et al. [10] reported, the development of systems which avoids the collision with pedestrians using the millimeter wave radar and the stereo camera is progressing. In the system development, Raksincharoensak et al. [11] reported the improvement in the detection accuracy of pedestrian's movement vector to predict a collision is one of the major research items. In addition to the improvement in the accuracy of pedestrian detection, the optimization of Human-Machine Interface in terms of the interaction between the driver and the system is a crucial issue to be discussed. The optimization of braking control timing is important so that drivers are not dependent on the system excessively and the trust on system is maintained. For example, as Itoh [12] reported, when the brake timing by the system is extremely late, the trust on the braking support system may decrease. Moreover, when the brake timing of the system is extremely early, a driver may depend on the system extremely. In a preceding study dealing with the design of control timing for collision-avoidance support brake system for avoiding the rear-end collision, Suzuki et al. $[13,14]$ have reported the concept of system design as a part of the research activity of Japanese ASV projects. In this research, the braking by drivers for avoiding rear-end collision was analyzed in the driving simulator investigations as well as the experiments on the test course, and a braking control timing which does not cause the excessive dependence on the system of drivers was proposed. In this study, it was reported that to minimize the interference between braking operation by driver and the braking control by the system is important for the avoidance of excessive dependence on the system. In this research, the design method of brake timing of the systems which avoids the collision with a pedestrian is analyzed. First, the braking operation timing by drivers, when a pedestrian comes out from a sidewalk, is analyzed using a driving simulator. Subsequently, based on the results of this investigation for quantifying the braking operation timing of drivers, a design method of braking control timing is shown in consideration of the following two conditions:

(1) There is no excessive interference between the braking controlled by the systems and the braking operated by the drivers, and the driver may not depend on the system excessively;

(2) The system starts braking control early rather than the brake timing which is a physical limit of collision avoidance, and the trust on the system is maintained.

\section{Investigation of Brake Timing of Drivers}

\subsection{Experimental Condition}

In a virtual space of driving simulator in Kagawa University, the conditions when a pedestrian comes out to a road from the sidewalk were set up. This experimental scenario is depicted in Fig. 1. As for the pedestrian's crossing velocity ( $\mathrm{Vp}$ ), two conditions, 5 $\mathrm{km} / \mathrm{h}$ and $8 \mathrm{~km} / \mathrm{h}$ were set up. Three different experiment conditions for the vehicle velocity of a subject vehicle (V) which are $20 \mathrm{~km} / \mathrm{h}, 40 \mathrm{~km} / \mathrm{h}, 60$ $\mathrm{km} / \mathrm{h}$ were set up. The condition on which a pedestrian comes out to the road from the sidewalk at the timing of "Time to Collision (TTC; relative distance[m]/relative velocity $[\mathrm{m} / \mathrm{s}])=3 \mathrm{~s}$ " was set. That is, the timing to which a pedestrian begins to come out to the road was set so that TTC might become the same on all conditions. In this experiment scenario, when only a risk event of a pedestrian coming out to the road from the sidewalk was set, the tendency for subject drivers to become sensitive at a risk event was observed. For this reason, not only one risk event but also nine fake events, like a pedestrian suddenly stops just before coming out to a road or suddenly change the direction of movement and does not cross a road were included. This means that each subject driver experienced sixty events in total including six risk events. The experimental 


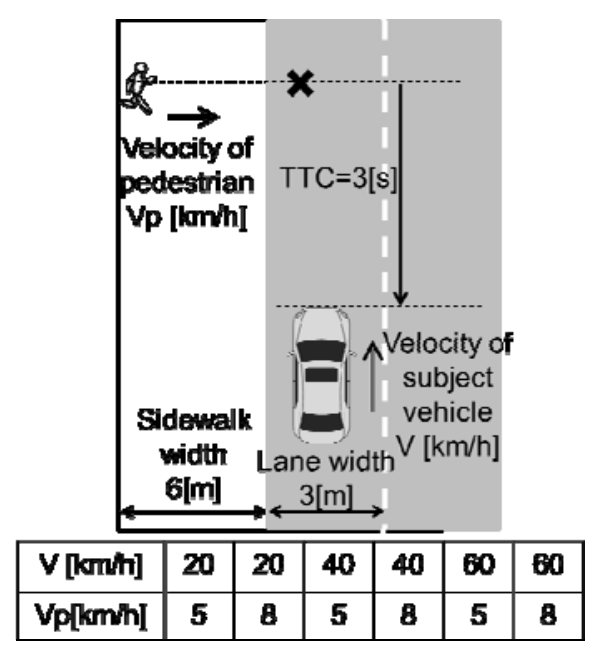

(a) A set-up for evaluating braking behavior to avoid the collision with pedestrian

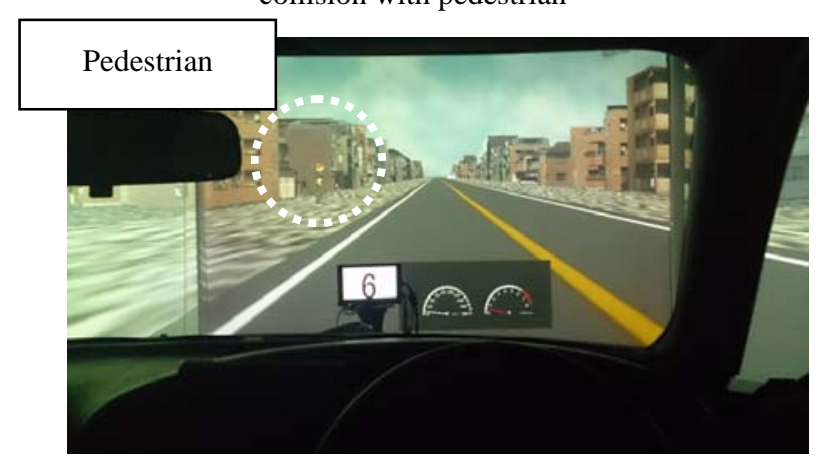

(b) A driving scene in a driving simulator

Fig. 1 Experimental scenario.

Table 1 Experimental sequence.

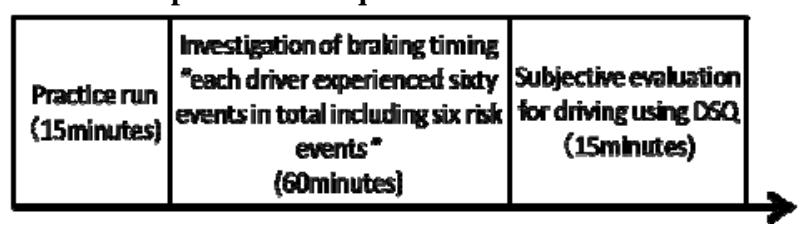

sequence is shown in Table 1 . We focused on these six events and investigated the driver behavior. In a prior experiment, it was observed that subject drivers became sensitive to a pedestrian, which is different from the actual driving behavior. Therefore, the numbers on an LCD display which is mounted on the instrument panel were presented every $3 \mathrm{~s}$, and the mental calculation was set to a subject driver as a mental distraction. In this experimental study, ten male drivers participated as the subject drivers. An average and standard deviation of the age of subject drivers were $22.4 \pm 0.86$ years old. In starting this experiment, the validity of the contents of experiment was verified by an experiment-ethics committee of Kagawa University. In addition, each of the experiment participants got informed consent from the experiment conductor before the experiment started. In this study, only young male drivers participated in the experiments. An additional study including female drivers or senior drivers as test subjects will be needed. In this study, we will propose a concept for designing the braking onset timing of the system and verify the validity of this concept which was mentioned at (1) and (2) in a previous section.

\subsection{Investigation Results of Braking Operation of Drivers}

The average of braking operation start timing of subject drivers in terms of TTC is shown in Fig. 2 for each velocity of subject vehicles. The analysis regarding the variance of braking operation timing among each condition based on ANOVA is shown in Table 2. This table shows that there is no big difference for braking behavior among each driving condition in terms of the crossing velocity of pedestrian and velocity of subject vehicle. In this experimental study, the drivers operated braking at approximately $2.1 \mathrm{~s}$ before colliding with the pedestrian. This means that driver started braking suddenly after a pedestrian appeared at the timing of $3.0 \mathrm{~s}$ before colliding to the subject vehicle.

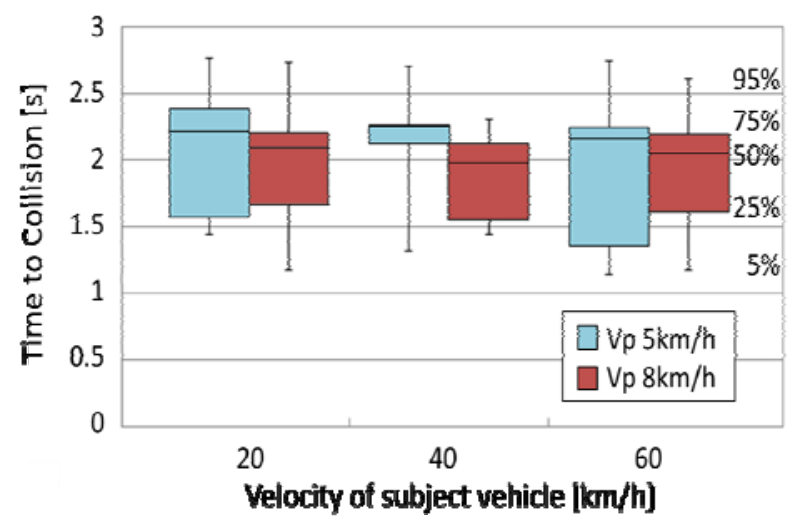

Fig. 2 The braking operation timing of subject drivers for each vehicle velocity. 
Table 2 The variance of braking operation timing among each condition.

(a) ANOVA results when $\mathrm{Vp}=5 \mathrm{~km} / \mathrm{h}$

\begin{tabular}{|c|c|c|c|c|c|c|}
\hline $\begin{array}{l}\text { Source of } \\
\text { Variation }\end{array}$ & SS & $d f$ & MS & $F$ & $P$-value & F crit \\
\hline $\begin{array}{l}\text { Between } \\
\text { Groups }\end{array}$ & 0.081103 & & 0.040551 & 0.229775 & 0.79644 & 3.402826 \\
\hline $\begin{array}{l}\text { within } \\
\text { Groups }\end{array}$ & 4.235597 & & 0.176483 & & & \\
\hline Total & 4.3167 & 26 & & & & \\
\hline \multicolumn{7}{|c|}{ (b) ANOVA results when $V p=8 \mathrm{~km} / \mathrm{h}$} \\
\hline $\begin{array}{l}\text { Source of } \\
\text { Variation }\end{array}$ & SS & $d f$ & MS & $F$ & $P$-value & F crit \\
\hline $\begin{array}{c}\text { Between } \\
\text { Groups }\end{array}$ & 0.01924 & 2 & 0.00962 & 0.086162 & 0.917728 & 3.402826 \\
\hline $\begin{array}{l}\text { within } \\
\text { Groups }\end{array}$ & 2.679585 & & 0.111649 & & & \\
\hline Total & 2.698825 & 26 & & & & \\
\hline \multicolumn{7}{|c|}{ (c) ANOVA results among all groups } \\
\hline $\begin{array}{l}\text { Source of } \\
\text { Variation }\end{array}$ & SS & $d f$ & MS & $F$ & $P$-value & $F$ crit \\
\hline $\begin{array}{l}\text { Between } \\
\text { Groups }\end{array}$ & 0.219872 & 5 & 0.043974 & 0.305237 & 0.907382 & 2.408514 \\
\hline $\begin{array}{l}\text { within } \\
\text { Groups }\end{array}$ & 6.915182 & 48 & 0.144066 & & & \\
\hline Total & 7.135054 & 53 & & & & \\
\hline
\end{tabular}

In preceding researches by Suzuki et al. [13, 14], when the velocity of subject vehicle is higher, the brake timing of the drivers for avoiding rear-end collision with a leading vehicle becomes earlier. This means that the driver operated braking in taking account of total stopping distance to avoid rear-end collision. One of the experimental results by Suzuki is shown in Fig. 3. When the velocity of the vehicle is higher, the timing of braking became earlier. In this figure, the TTC which considers the total stopping distance to avoid the rear-end collision with a leading vehicle is also shown. It is possible to say that the TTC regarding the starting of braking by drivers became larger related to the increase of this TTC which considers the total stopping distance to avoid the collision.

In the experiment to avoid the collision with a pedestrian, the experimental results differ from this tendency to avoid the rear-end collision. For example, in this experiment scenario of which a pedestrian

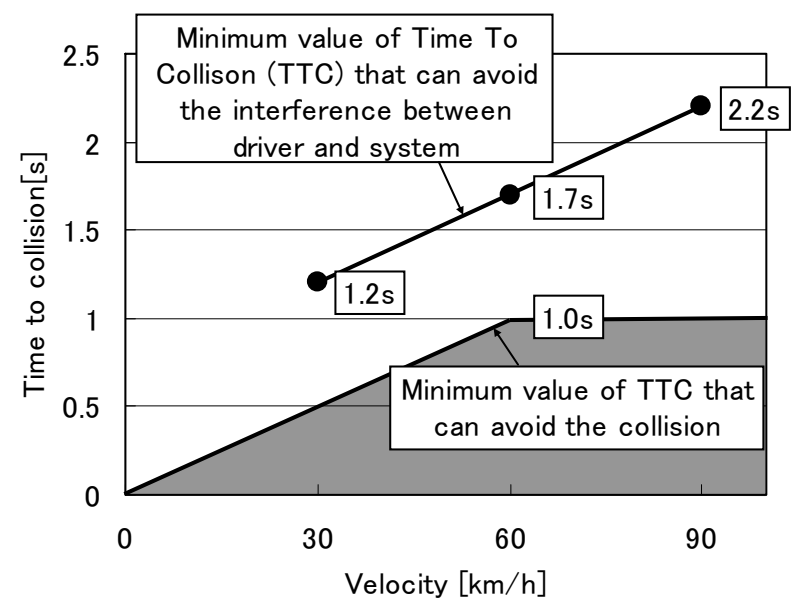

Fig.3 The brake timing of the drivers for avoiding rear-end collision with a leading vehicle $[13,14]$.

comes out to the road from the sidewalk, the different braking operation timing depending on the velocity of subject vehicle ("V" in Fig. 1) or crossing velocity of pedestrian ("Vp" in Fig. 1) was not observed. That is, in the risk scenario in which a pedestrian comes out from the sidewalk to the road, after the subject driver had recognized that a pedestrian comes out to the road at three seconds of TTC, the subject drivers started braking operation after approximately same latency regardless of the velocity of subject vehicle and the crossing velocity of pedestrian.

Next, about all the subjects' braking operation timing shown in above-mentioned Fig. 2, the cluster analysis of driving behavior using DSQ (Driving Style Questionnaire) [15] was conducted and the driver group was classified into three groups. The driver characteristics of these three groups with eight mental characters are shown in Fig. 4 based on the evaluation index in DSQ. We will look at the relation between the characteristics of each driver group and onset timing of braking behavior.

Three different average brake timing of each subject group in terms of TTC is shown in Fig. 5. Only subject drivers classified into the group \#1 is in the tendency for braking start timing to be delayed extremely. When we look at the mental character of subject drivers classified into this group \#1, this driver group has hasty driving character and worrying character, as shown in 


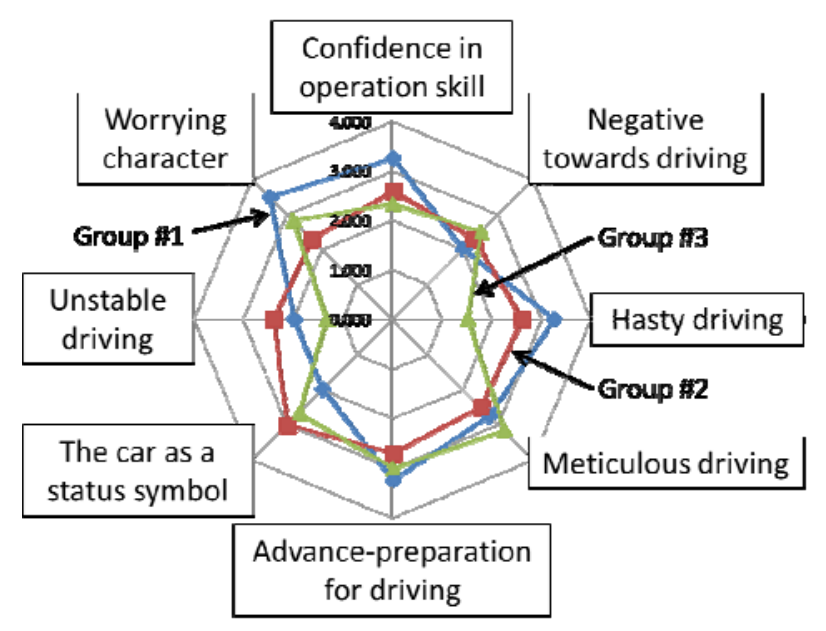

Fig. 4 Analysis result of driver characteristics by DSQ.

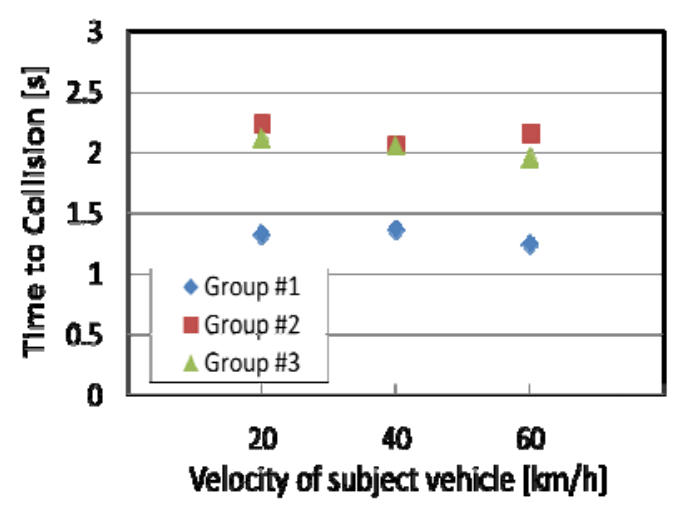

Fig. 5 Average value of braking start timing of each driver group.

Fig. 4. That is, as for the drivers classified into a hasty tendency, its brake operation timing tends to be delayed and TTC at braking becomes short. Regarding the drivers classified into group\#2 or \#3, these driver groups do not have hasty driving character so that the braking onset timing is relatively early. Though it is not possible to explain the relation between the driving mental characteristics and onset timing of braking behavior in detail, we can grasp the tendency between them by DSQ.

\section{Method for Designing the Braking Control Timing}

\subsection{Design of Braking Control Timing}

In designing braking control timing, the following requirements were determined in this study.
(1) The braking control timing of a system is not dependent on the velocity of subject vehicles, and is set up uniformly. It is because a significant difference was not observed for the braking start timing of subject drivers on all condition of velocity.

(2) On all the velocity conditions (20 km/h, $40 \mathrm{~km} / \mathrm{h}$ and $60 \mathrm{~km} / \mathrm{h}$ ), the system can avoid the collision with a pedestrian completely.

An example of TTC which satisfies the above conditions is $1.3 \mathrm{~s}$ and shown in Fig. 6. In the frequency distribution in terms of braking initiation timing, $1.3 \mathrm{~s}$ of TTC is equivalent to approximately 5 percentiles in whole frequency distribution of the brake timing as shown at Fig. 2 and equivalent to the average of braking timing in group\#1 which showed most delayed timing. This means that $95 \%$ of braking initiation timing in terms of TTC is longer than $1.3 \mathrm{~s}$ among all subject drivers. The braking operation by drivers when the driver used the system is discussed in the following experiments.

\subsection{Interference of Braking Operation by Driver and Braking Control by System}

As for the vehicle velocity of the car which a subject drives, the experiment was conducted on a condition of $20 \mathrm{~km} / \mathrm{h}, 40 \mathrm{~km} / \mathrm{h}$ and $60 \mathrm{~km} / \mathrm{h}$. The braking timing at $1.3 \mathrm{~s}$ of TTC and three different timing $(0.45 \mathrm{~s}$ for $20 \mathrm{~km} / \mathrm{h}, 0.77 \mathrm{~s}$ for $40 \mathrm{~km} / \mathrm{h}, 1.1 \mathrm{~s}$ for $60 \mathrm{~km} / \mathrm{h}$ ) related to the velocity of subject vehicle which is a physical

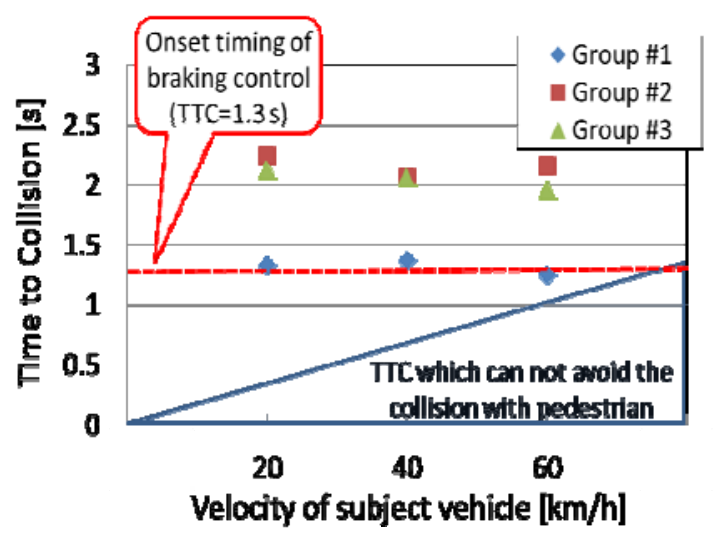

Fig. 6 Average of braking start timing of each driver group. 
limit to avoid the collision with a leading vehicle was set up. The timing of $1.3 \mathrm{~s}$ is equivalent to above mentioned timing which may avoid the excessive interference between the system operation and driver braking. The physical limit is a most delayed timing that is a limit to avoid the collision to a leading vehicle. One risk event and nine fake events were set as same as the above mentioned experiment for investigating drivers' brake timing. About a pedestrian’s crossing velocity, two conditions were set. That is, the subject driver experienced twenty events including two risk events. Six subject drivers participated in the experiment.

One example of time sequence of braking operation by driver and braking control by the system is shown in Fig. 7. This figure shows the braking behavior of

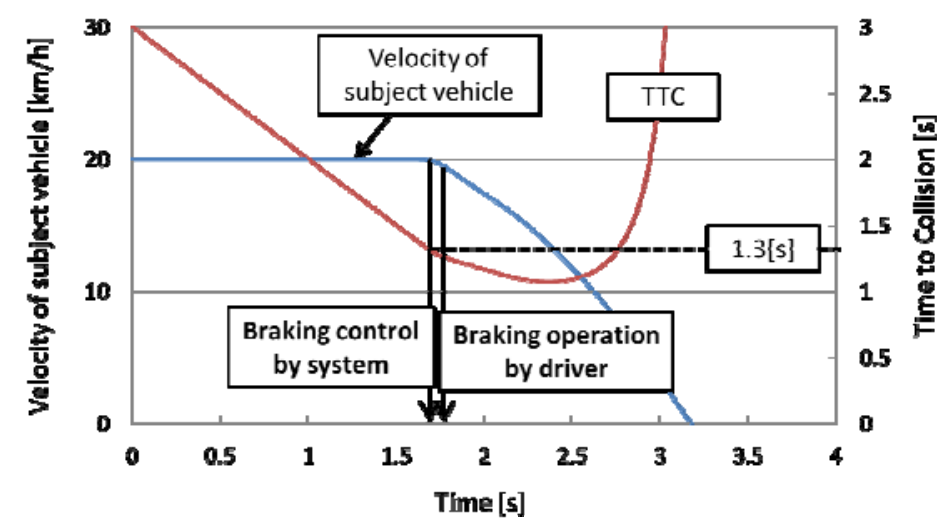

(a) Velocity of subject vehicle: $20 \mathrm{~km} / \mathrm{h}$

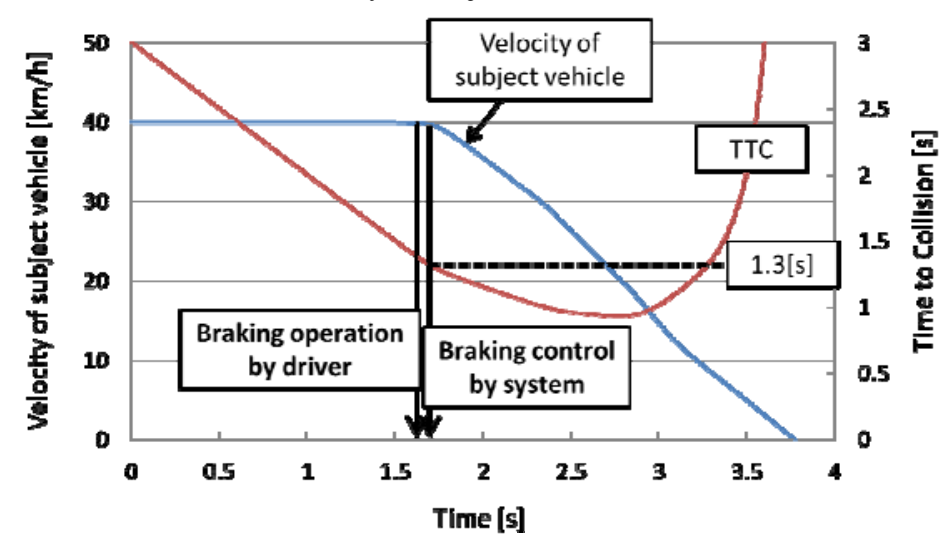

(b) Velocity of subject vehicle: $40 \mathrm{~km} / \mathrm{h}$

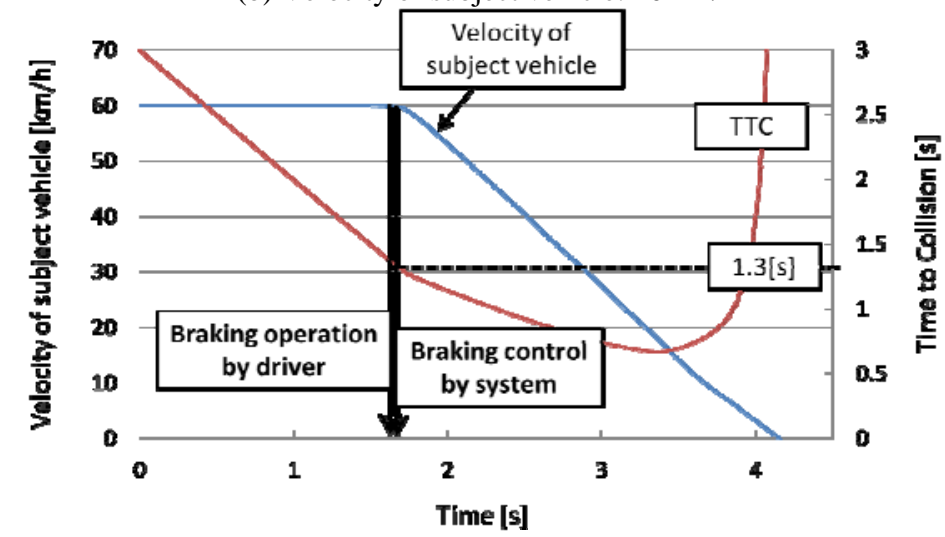

(c) Velocity of subject vehicle: $60 \mathrm{~km} / \mathrm{h}$

Fig. 7 Time sequence of braking operation by driver whose braking timing is extremely late classified into group\#1 and braking control by the system. 
group\#1 whose braking timing is extremely late. As shown in Fig. 7, the braking timing by the system is not extremely earlier than braking timing by driver and almost equal to the braking timing by the system. Even for the driver who was classified into the group\#1 whose braking initiation is extremely late, the excessive interference between the system and driver was not observed. Regarding the braking behavior of group\#2 or \#3 whose braking timing is earlier than the braking timing of this group\#1, the braking timing by driver was much earlier than the braking control by the system and showed no interference between the braking control by the system and braking by the driver. The subjective evaluation showed that it was possible to keep the system trust high and dependence on the system low simultaneously, when the onset timing of the system is set to $1.3 \mathrm{~s}$ of TTC. We will continue an additional study by more subject drivers to validate these results.

Now, we will look at the subjective evaluation regarding the system trust. The results of all subjective drivers are shown in Fig. 8. This figure gives us following remark. The trust on the system control when the braking started at $1.3 \mathrm{~s}$ of TTC is higher than the trust when the braking started at the physical time limit to avoid the collision. We will continue the analysis regarding the relation between the braking control timing by the system and the trust on the system.

\section{Discussion}

\subsection{Major Factors for the Start Timing of Braking Behavior}

In this study, the different braking operation timing to avoid the collision with a pedestrian depending on the velocity of subject vehicle or crossing velocity of pedestrian was not observed. This tendency in terms of avoiding a collision differs from that of avoiding a rear-end collision to a leading vehicle. Then, we analyze the reason why drivers operated braking at almost same timing of TTC. We conducted an additional experiment under the conditions which

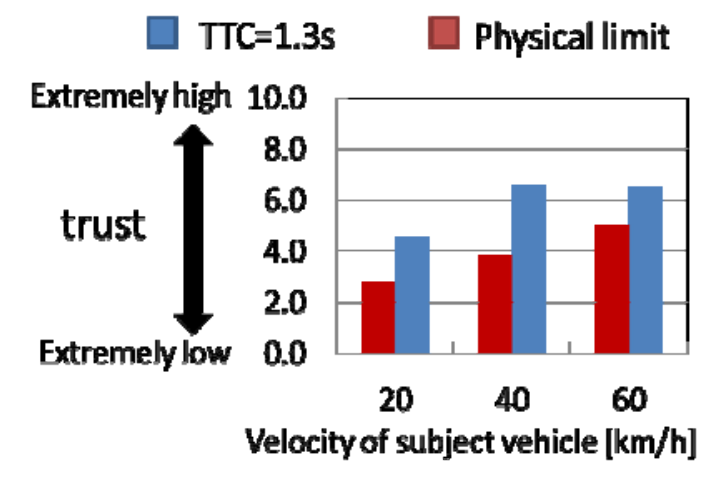

Fig. 8 The subjective evaluation regarding the system trust and annoyance of the system control.

changed the appearance timing of a pedestrian in terms of TTC. In this additional experiment, the pedestrian's appearance timing was set at 4.5 s of TTC, which is longer than that of main experiment mentioned above.

In this experiment, pedestrian's crossing velocity of pedestrian (Vp) was only one condition with $6 \mathrm{~km} / \mathrm{h}$ which is same as one of two conditions in the above-mentioned experiment. In this experiment, it was set up that the pedestrian appears at $4.5 \mathrm{~s}$ of TTC, which is $1.5 \mathrm{~s}$ earlier than the timing of main experiments which was mentioned at previous section. This means that the pedestrian appeared from the more distant point from a driveway. The velocity of subject vehicle $(\mathrm{V})$ of three conditions was set up also in this additional experiment. Also in this experiment, the fake events were set up. That is, a pedestrian appeared at TTC of $4.5 \mathrm{~s}$ and then stopped just before coming out to the road, in order to prevent simple reaction of drivers. The experimental result in this condition is shown in Fig. 9.

The timing of braking operation of drivers showed almost same value, though the velocity of vehicles changed. This tendency is same as the main experiment in which the pedestrian appeared at the timing of $3.0 \mathrm{~s}$ of TTC. On the conditions in which the pedestrian appeared in the above-mentioned TTC $=3.0 \mathrm{~s}$, the median (50\%ile) of braking operation timing was about $2.1 \mathrm{~s}$. On the conditions in which the pedestrian appeared at $4.5 \mathrm{~s}$ of TTC, the median (50\%ile) of 


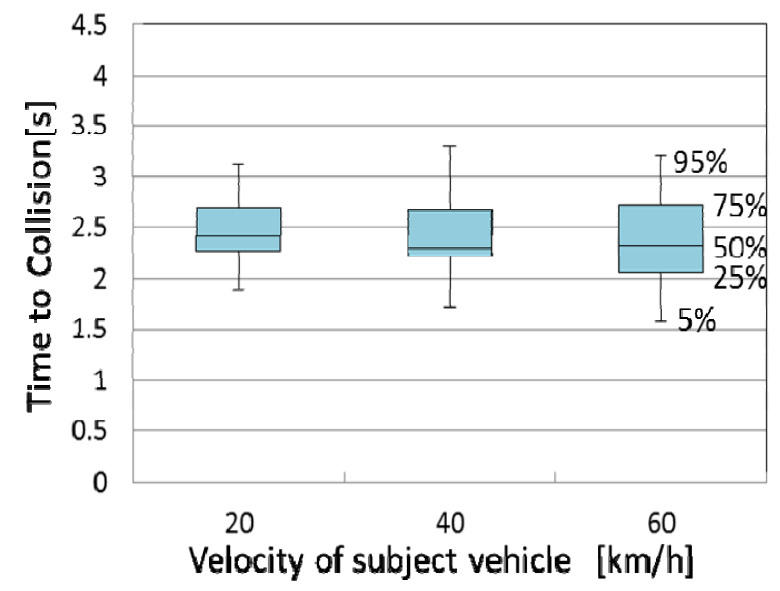

Fig. 9 The braking operation timing of subject drivers for each vehicle velocity (appearance timing of pedestrians: TTC = $4.5 \mathrm{~s}$ ).

braking operation timing was about $2.4 \mathrm{~s}$. In the event that a driver avoids a pedestrian who runs out from a sidewalk, it turned out a driver started braking at almost same timing regardless of the velocity of subject vehicle. Furthermore, the following tendencies are clear when this experimental result is analyzed in detail. Under the conditions in which the pedestrian appeared at $3.0 \mathrm{~s}$ of TTC, the time latency for braking to avoid the collision to a pedestrian is approximately $0.9 \mathrm{~s}$. That is, it can be judged that after recognizing a pedestrian, the driver has started braking operation immediately. When the pedestrian appeared at $4.5 \mathrm{~s}$ of TTC in this additional study, the braking latency from an appearance of pedestrian is approximately $2.1 \mathrm{~s}$. It turns out that time latency for braking operation by drivers to avoid the collision became longer because the risk of colliding with a pedestrian is lower. It is concluded that the timing of braking operation is not dependent on the velocity of subject vehicle, but is dependent on TTC related to the risk level of colliding with pedestrians. If the velocity of subject vehicle increases, the braking distance for fully stopping will be prolonged. But in the scenario of which a pedestrian runs out to a road from a sidewalk, it was relatively difficult to take this braking distance into consideration for drivers. As compared with the scenario in which a leading vehicle of the front of a driver started sudden braking, the braking behavior for driver was different at this scenario that a pedestrian goes out to a road, because it was difficult for drivers to estimate the total distance for avoiding the collision. We think that when detecting a pedestrian at the peripheral visual area of subject drivers, it was difficult to estimate the risk level of collision which can be explained with an appearance timing of pedestrian in terms of TTC and the stopping distance of subject vehicle simultaneously.

\subsection{Braking Control Timing by the System that Will Not} Interfere with the Braking Operation by the Drivers

In this study, we set up a situation that a pedestrian appeared at $3.0 \mathrm{~s}$ of TTC and $4.5 \mathrm{~s}$ of TTC and investigated the braking latency of the driver. As one of the results, we concluded that the start timing of braking operation depends on this appearance timing of the pedestrian in terms of TTC. This suggests that we should discuss what kind of timing is a typical timing of the appearance of pedestrians from a sidewalk. In this study, the velocity of a pedestrian is $5 \mathrm{~km} / \mathrm{h}$ and $8 \mathrm{~km} / \mathrm{h}$. Therefore, the distance between a lateral position of pedestrian and a lateral position where a subject vehicle runs is $4.16 \mathrm{~m}$ and $6.66 \mathrm{~m}$ in a situation that a pedestrian appeared at $3.0 \mathrm{~s}$ of TTC. When a pedestrian appears at $4.5 \mathrm{~s}$, this distance is $6.25 \mathrm{~m}$, under a condition that the walking velocity was set up as $5 \mathrm{~km} / \mathrm{h}$. These distances between a pedestrian and a road where a subject vehicle runs are depicted in Fig. 10a. It would be difficult to determine the distance from a pedestrian to a road that would be visible from the driver. But, we think the condition set up in this study is one of the typical in urban city area. One of the important objectives in this study is to clarify the shortest TTC when the drivers started braking to avoid the collision with pedestrians and clarify the onset timing of the system to minimize the interference between braking operations by drivers and braking control by the system. As we discussed that the braking latency of drivers was approximately 


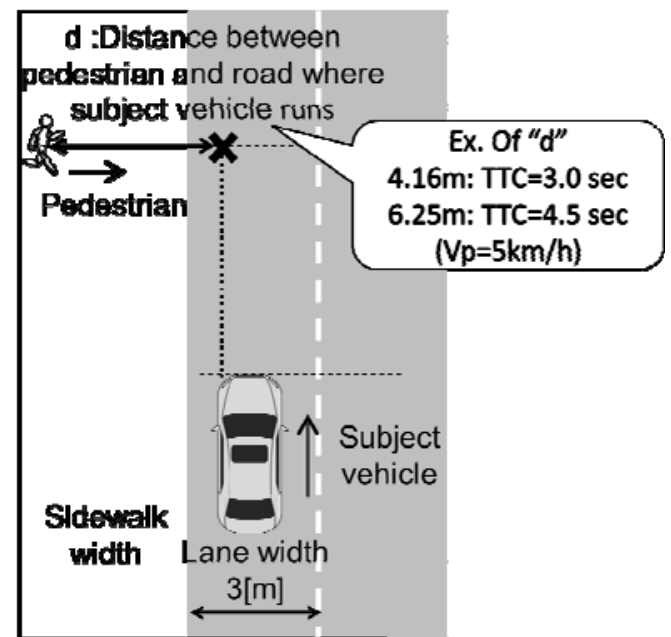

(a) The distance between a pedestrian and a road where a subject vehicle runs

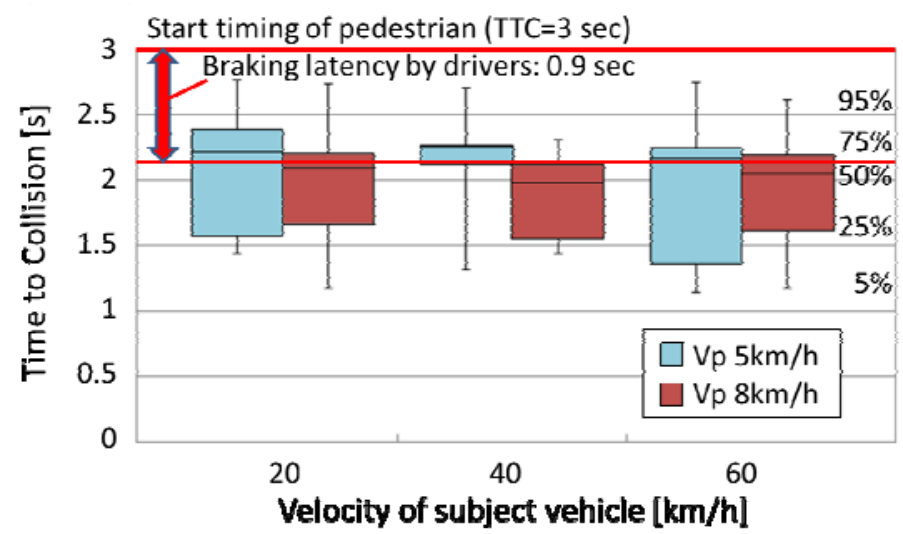

(b) The relation between a start timing of pedestrian and braking timing at three different velocity conditions

Fig. 10 The validation of the scenario in this study in terms of setting up a critical situation to avoid collision with pedestrian.

$0.9 \mathrm{~s}$ when a pedestrian appeared at $3.0 \mathrm{~s}$ of TTC. This means that we could set up one of the most risky driving situation and could analyze the shortest TTC of braking latency to avoid the collision with a pedestrian (Fig. 10b). Therefore, it can be concluded that the validity of results in this study for the designing of braking onset timing by the system is acceptable.

\section{Summary}

In this study, drivers' braking start timing to avoid collision with a pedestrian coming out from the sidewalk to the road was analyzed. It was observed that the drivers operated braking approximately at same timing in terms of TTC, though the velocity of a subject vehicle and crossing velocity of pedestrians are different. This tendency is different from that of avoiding rear-end collision to a leading vehicle. To clarify the reason why there is a difference among these braking behaviors is a next item of our study. In the frequency distribution in terms of braking initiation timing, $1.3 \mathrm{~s}$ of TTC was equivalent to five percentiles in whole frequency.

Based on the investigation of this braking behavior, the onset timing of braking control at $1.3 \mathrm{~s}$ of TTC was set up for all conditions of vehicle velocity and crossing velocity of pedestrians. The negative effects which are equivalent to the excessive dependence were not observed, when the system started braking control at the timing of $1.3 \mathrm{~s}$ of TTC, because there was no excessive interference between braking control by the system and braking operation by drivers. It was also observed that the system trust by drivers does not decrease at this control timing, because the brake 
timing by the system was not extremely late and was acceptable for the drivers.

In this study, we concluded that the timing of braking operation is not dependent on the velocity of subject vehicle, and is dependent on TTC related to the risk level of colliding with pedestrians. That means that the driver does not have enough sense for detecting the relation between the rest of time for colliding with a pedestrian and the rest of time for stopping vehicle. This braking behavior is not same with the behavior to avoid the rear-end collision to a vehicle ahead. We will continue the discussion why the difference was observed. One reasonable reason is that detecting pedestrians at the peripheral viewing area of driver required the higher mental workload to a driver than detecting a sudden deceleration of a leading vehicle at central viewing area. Therefore, the driver had difficulty to control the braking timing in consideration of the relation between the time colliding with a pedestrian and the time for stopping to avoid collision.

\section{References}

[1] Ishida, S., Sawamoto, K., Miyagawa, K., Nakanishi. Y., and Sasabuchi, Y. 2014. "Technical Introduction which Reduces the Traffic Accident by the Data Fusion of a Camera and a Millimeter Wave Sensor." Journal of Society of Automotive Engineers of Japan 68 (12): 31-7.

[2] Miichi, Y., and Nakagawa, H. 2014. "Approach for Development of Safe Driver Assistance Technology.” Journal of Society of Automotive Engineers of Japan 68 (12): 19-24.

[3] Ujihashi, S., and Kitaoka, T. 2014. "Evaluation of Active Safety Performance of Vehicle and Its Introduction to JNCAP.” Journal of Society of Automotive Engineers of Japan 68 (12): 12-8.

[4] Distner, M., Bengtsson, M., Broberg, T., and Jakobsson, L. 2009. "City Safety, a System Addressing Rear-End Collisions at Low Speeds.” In Proceedings of the 21st ESV Conference, Paper No. 09-0371.

[5] Coelingh, E., Jakobsson, L., Lind, H., and Lindman, M. 2007. "Collision Warning with Auto Brake, a Real-Life
Safety Perspective." In Proceedings of the 20th ESV Conference, Paper No. 07-0450.

[6] Brannstrom, M., Coelingh, E., and Sjoberg, J. 2010. "Model-Based Threat Assessment for Avoiding Arbitrary Vehicle Collisions." Transactions of Intelligent Transportation Systems, IEEE 11 (3): 658-69.

[7] Ljung-Aust, M., Jakobsson, L., Lindman, M., and Coelingh, E. 2015. "Collision Avoidance Systems-Advancements and Efficiency.” SAE Technical Paper 2015-01-1406, doi:10.4271/2015-01-1406.

[8] Isaksson-Hellman, I. 2012. "The Effect of a Low-Speed Automatic Brake System Estimated From Real Life Data.” Transaction of Association for the Advancement of Automotive Medicine 56: 231-40.

[9] Inomata, R., Hayashi, H., Fujishiro, R., Ouchi, Y., Nanami, T., and Suzuki, K. 2014. "Development of Pre-crash Safety System with Collision Avoidance Assist.” Journal of Society of Automotive Engineers of Japan 68 (12): 25-30.

[10] Coelingh, E., Eidehall, A., and Bengtsson, M. 2010. “Collision Warning with Full Auto Brake and Pedestrian Detection-A Practical Example of Automatic Emergency Braking." In Proceedings of Intelligent Transportation Systems (ITSC), 13th International IEEE Conference, 155-60.

[11] Raksincharoensak, P., Sakai, Y., and Shimizu, I. 2014. "Development of Pedestrian Collision Avoidance System Based on Crosswalk Recognition by an On-board Camera." Transactions of Society of Automotive Engineers of Japan 45 (2): 375-80.

[12] Itoh, M. 2012. "Toward Overtrust-Free Advanced Driver Assistance Systems.” Cognition, Technology \& Work 14 (1): 51-60.

[13] Suzuki, K., and Marumo, Y. 2002. “A Study on the Onset Timing of Collision Avoidance Assistance Systems for Minimizing the Over-reliance on the System." Transactions of the Japan Society of Mechanical Engineers 69 (688 C): 96-102.

[14] Suzuki, K., and Marumo Y. 2003. "Study on the Control Algorithm of Driving Assistance System to Inhibit the Over-dependence on the System.” Transactions of the Japan Society of Mechanical Engineers 70 (699 C): 293-9.

[15] Ishibashi, M., Okuwa, M., Doi, S., and Akamatsu, M. 2008. "Characterizing Indices of Driving Style and Their Relevance to Car Following Behaviour.” Transactions of Society of Automotive Engineers of Japan 39 (1): 121-6. 